\title{
FACTORING POINTLIKE SIMPLICIAL MAPPINGS
}

\author{
BY \\ C. L. WIGINTON
}

I. Introduction. An outstanding question in topology is, "If $D$ is a decomposition of a space $X$, under what conditions is the decomposition space homeomorphic to $X$ "? Much has been done toward answering this question and in the process several problems, interesting in themselves, have arisen. The following belongs to this category.

A mapping from the $n$-sphere onto a space $X$ is pointlike provided the complement of the inverse image of each point of $X$ is homeomorphic to $n$-space. The star of a simplex $\sigma$ is the union of all simplexes which have $\sigma$ as a face. A mapping will be called a 1-Star collapse mapping provided the mapping is simplicial and identifies exactly two vertices, those bounding a 1 -simplex. Such a mapping takes the star of the 1-simplex to an $(n-1)$-cell.

In 1963, Ross Finney answered a special case of the above question [1]. He proved that if there exists a pointlike simplicial mapping from a 3-sphere onto a triangulated space $T$, then $T$ is a 3 -sphere. In his paper he asked if a pointlike simplicial mapping of the 3-sphere which preserves orientation could be factored into a sequence of 1-Star collapse mappings of the 3-sphere onto itself. This is certainly a natural way to visualize such mappings. The purpose of this investigation is to answer this question in the negative and to prove a necessary and sufficient condition for such factorization.

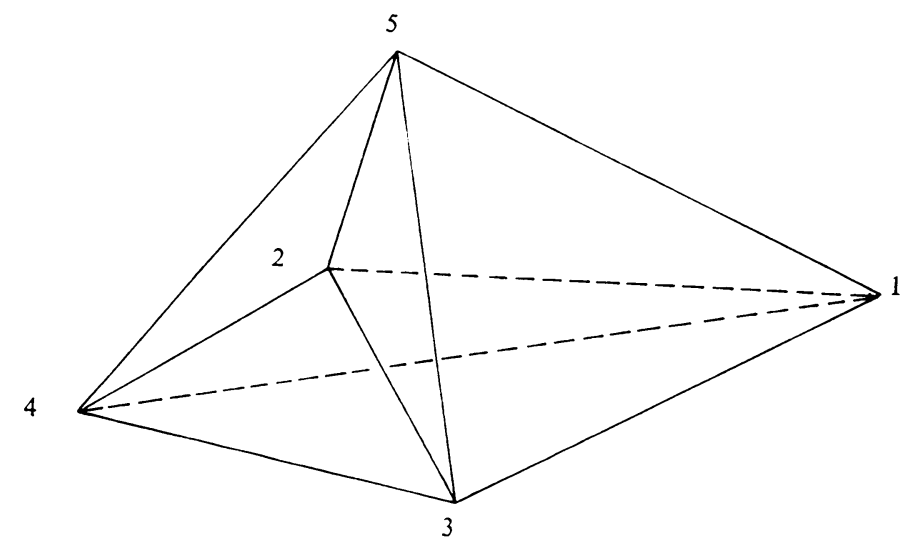

Figure 1.

Received by the editors May 13, 1965. 
II. Certain pointlike simplicial mappings cannot be factored. The proof that certain pointlike simplicial mappings cannot be factored into a sequence of 1-Star collapse mappings will be accomplished by the following:

(i) The triangulation of the domain 3-sphere will be described.

(ii) The triangulation of the range 3-sphere will be described.

(iii) The mapping $f$ will be defined.

(iv) The mapping $f$ will be shown to be pointlike and simplicial.

(v) It will be shown that a 1-Star collapse mapping cannot be defined on any 1-simplex mapped to a point by the mapping $f$ and have the 3-sphere as the range. Therefore it will not be possible to represent $f$ as a sequence of 1-Star collapse mappings of the 3-sphere onto itself.

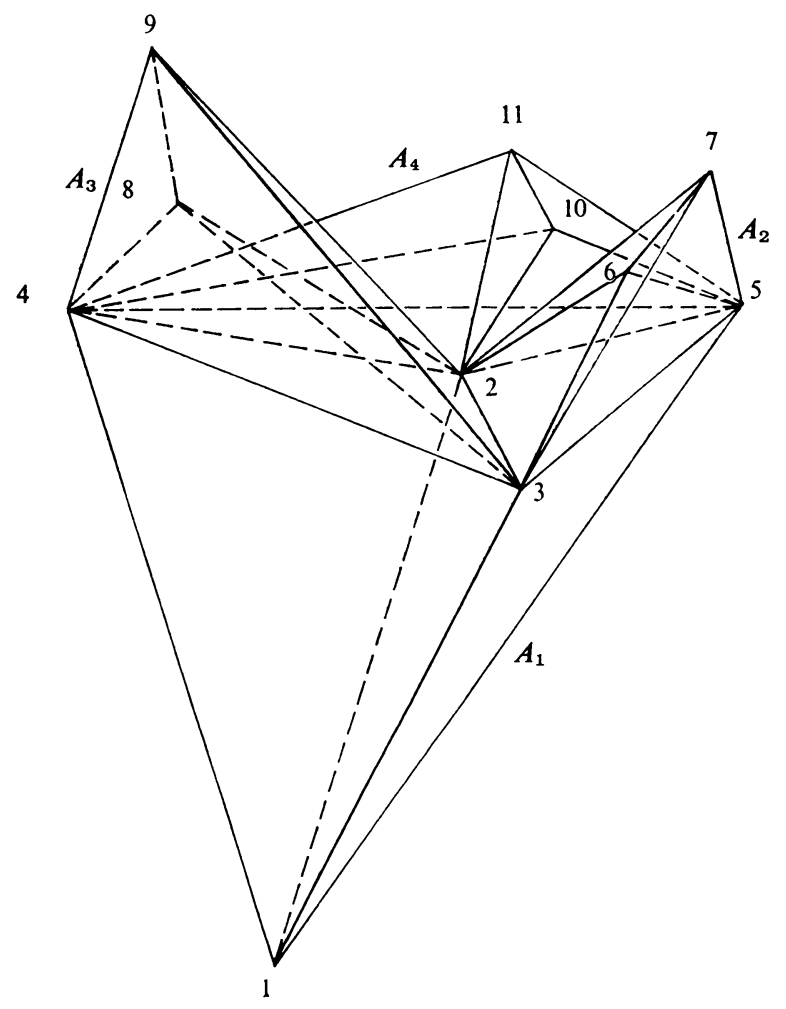

FIGURE 2.

Following this example a parallel question in dimension 3 will be answered.

(i) Triangulation of the domain 3-sphere. Let $A$ denote the union of three 3simplexes, each pair of which have a common 2-face. Numbers will be used to denote vertices. Consider four copies of the set $A$ with faces identified as shown in Figure 2, defining 3-simplexes as indicated. 
Let $\left[A_{2} A_{3}\right]$ denote the union of nine 3-simplexes with faces identified as shown in Figure 3.

This set connects $A_{2}$ to $A_{3}$ and is attached to the simplexes in Figure 2, as indicated by the names of the vertices. The numbers in parentheses serve to define sets $\left[A_{3} A_{4}\right]$ and $\left[A_{4} A_{2}\right]$, respectively, which are also attached to the simplexes in Figure 2 , as indicated by the names of the vertices.

Let $(2,7,9,11)$ define a simplex. (This "fills up" the top of the above collection.)

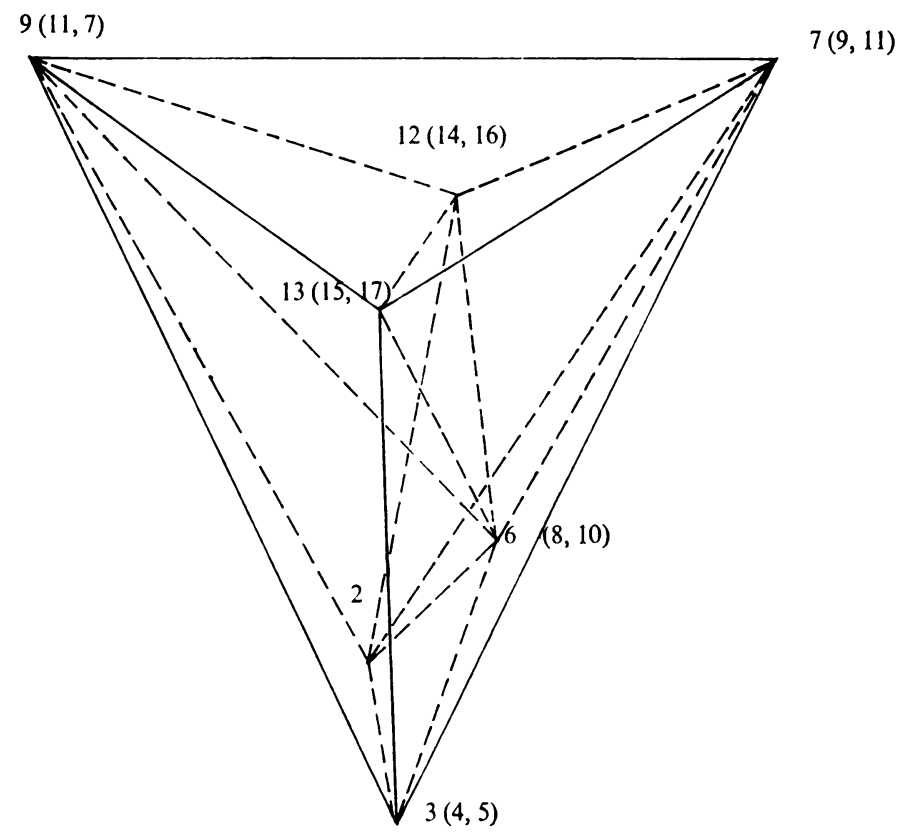

FIGURE 3.

Consider the collection of 3-simplexes shown in Figure 4.

This completes the star of $(3,4)$ and $(3,5)$ and connects between the stars of these 1-simplexes. Identification is again indicated by vertex numbering and the numbers in parentheses serve to define similar additions for the star of $(4,5)$ and to fill in between completions.

The exterior 2-simplexes of the complex thus described form a 2-sphere $S^{2}$ bounding a 3-cell $C_{3}$. The join of this set with a vertex, 24, gives a triangulated 3-sphere, $S^{3}$. This completes the description of the domain.

(ii) Triangulation of the range 3-sphere. A triangulated 3-sphere will now be described which will be the range of a simplicial mapping defined on the 3-sphere described above. Vertices 2, 3, 4, and 5 of the above 3-sphere will be identified under the mapping while no other pair of vertices is identified. Therefore in describing the range the numbers $3,4,5$ will not be used so that the mapping can be defined to take vertex $i$ to vertex $i^{*}$ for $i \neq 3,4,5$ and to take 3,4 , and 5 to $2^{*}$. 

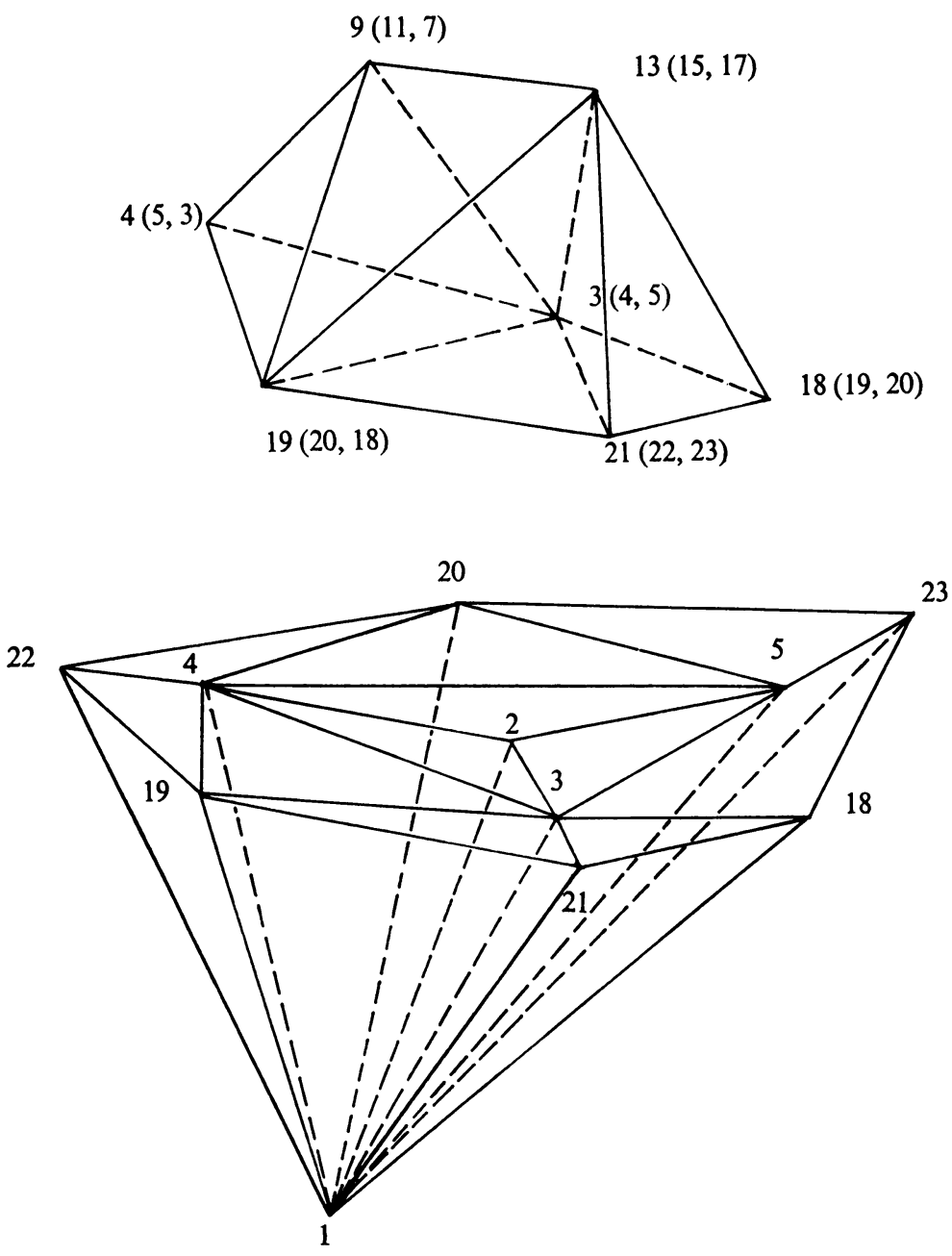

FIGURE 4.

Consider the collection of 3-simplexes in Figure 5.

As before numbers in parentheses serve to define two similar collections of 3simplexes and the adjoining of the sets is indicated by vertex names. The union of the above three sets and $\left(2^{*}, 7^{*}, 9^{*}, 11^{*}\right)$ begins the triangulation of the range.

Additional 3-simplexes are to be added to the simplexes of Figure 5 in the fashion shown in Figure 6.

This total collection of simplexes defines a 3-cell which, when joined with a vertex 24*, gives a 3-sphere. This completes the description of the range 3-sphere.

(iii) Definition of the mapping. On the vertices of the domain 3-sphere define

$$
\begin{aligned}
f_{v}(i) & =i^{*}, & & i \neq 3,4,5, \\
& =2^{*}, & & i=3,4,5 .
\end{aligned}
$$


It is easy to see that if a collection of vertices in the domain determines a simplex then the images determine a simplex in the range. Therefore it is possible to define a mapping $f$ to be the linear extension of $f_{v}$.

(iv) The mapping $f$ is pointlike and simplicial. The mapping is simplicial since it is defined to be the linear extension of a vertex mapping.

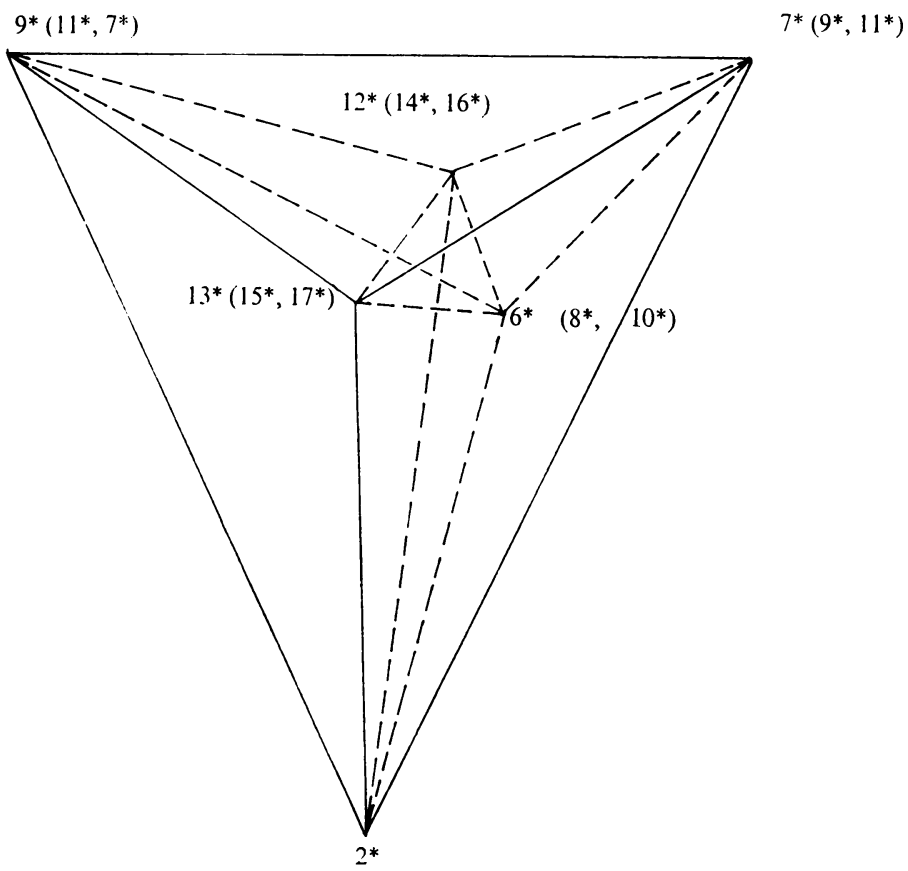

Figure 5.

It is easy to determine that the mapping is pointlike. It is the identity on the complement of the 3-cell, $C_{3}$. In $C_{3}$ certain 3-simplexes are mapped to 2-simplexes and hence decomposed into straight line segments. The only 3-simplexes affected in any other manner are in the sets $A_{i}$. The situation on these sets is illustrated in Figure 7. Therefore, if $p$ is a point interior to a 3-simplex, $f^{-1}(p)$ is a point. If $p$ is interior to a 2-simplex then $f^{-1}(p)$ is either a point or a polygonal arc. If $p$ is interior to a 1-simplex then $f^{-1}(p)$ is either a point, a line segment, or a triangle. If $p$ is a vertex then $f^{-1}(p)$ is either a vertex, a 1-simplex, or the disk composed of the three 2-simplexes mapping to $2 *$.

Since the inverse image of any point is a pointlike set, the mapping is pointlike.

(v) The mapping $f$ cannot be factored.

Lemma 0. If the set $A$ is part of a triangulation of a 3-sphere, $S^{3}$, then a 1-Star collapse mapping cannot be defined on $(3,4)$ and have the range be a 3-manifold without boundary. 


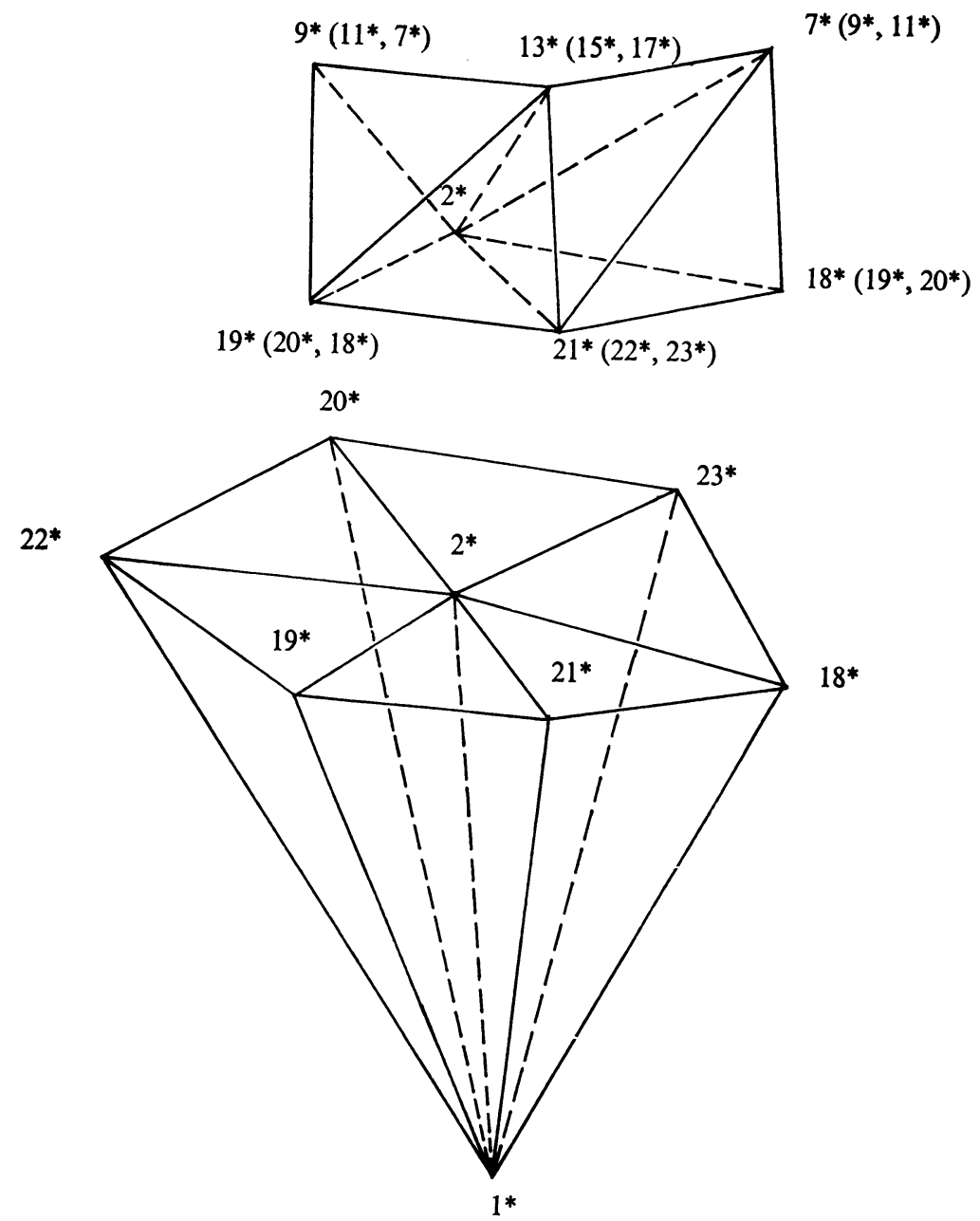

FIGURE 6.

Proof. It will be shown that there is no simplicial mapping of $S^{3}$ onto any 3manifold without boundary which identifies vertex 3 and vertex 4 while identifying none of 1,2 , and 5 .

Suppose such a mapping, $g$, exists. Then $g(1,2,5)$ is a 2 -simplex and hence the common face of exactly two 3-simplexes of the range. The only possible candidates for such 3-simplexes in the range are the images of $(1,2,4,5)$ and $(1,2,3,5)$ since only these 3 -simplexes have $(1,2,5)$ as a common face in the domain. However, the images of the sets of vertices $\{1,2,4,5\}$ and $\{1,2,3,5\}$ are the same and hence cannot determine distinct 3-simplexes. This contradiction shows that no such simplicial mapping can exist.

The simplex $(3,4)$ could have been replaced by $(4,5)$ or $(3,5)$ in the lemma and have the same conclusion due to symmetry. 
Since each 1-simplex mapped to a point by $f$ is one of the type described in the lemma, it is clear that it is impossible to represent $f$ as a sequence of 1-Star collapse mappings.

Once this question is settled in the negative it can be asked, "Is it possible to factor an orientation preserving pointlike simplicial mapping of $S^{3}$ into a sequence of simplicial mappings of $S^{3}$ onto itself each of which collapses either a 1-simplex or a 2-simplex?" A negative answer can be obtained from considering the preceding example. As noted the above mapping cannot be factored into simplicial mappings each of which collapses one 1-simplex. Since only three 2-simplexes are collapsed it is easy to see that this latter type factorization is also not possible. This is true since if a single 2-simplex, say $(2,3,5)$, were collapsed by a simplicial mapping of the 3-sphere onto itself then the 1-simplex $(2,5)$ would be collapsed while none of $\{4,10,11\}$ was identified. This, however, is not possible since this
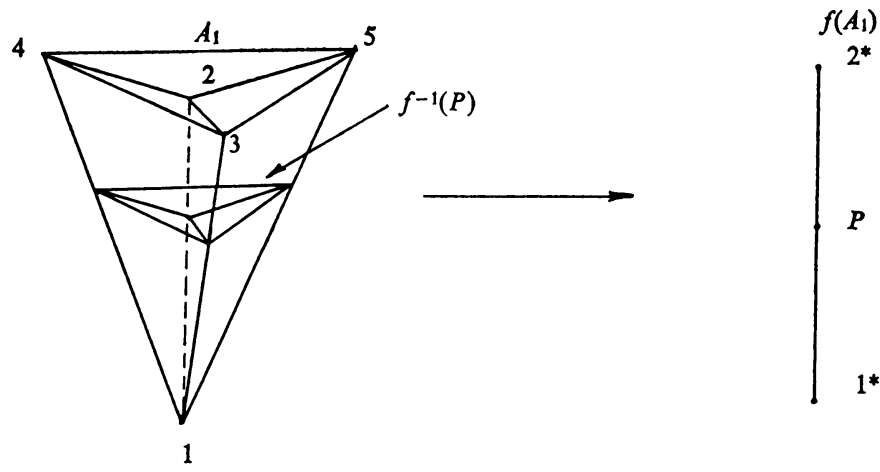

$A_{i}, \quad i=1,2,3$
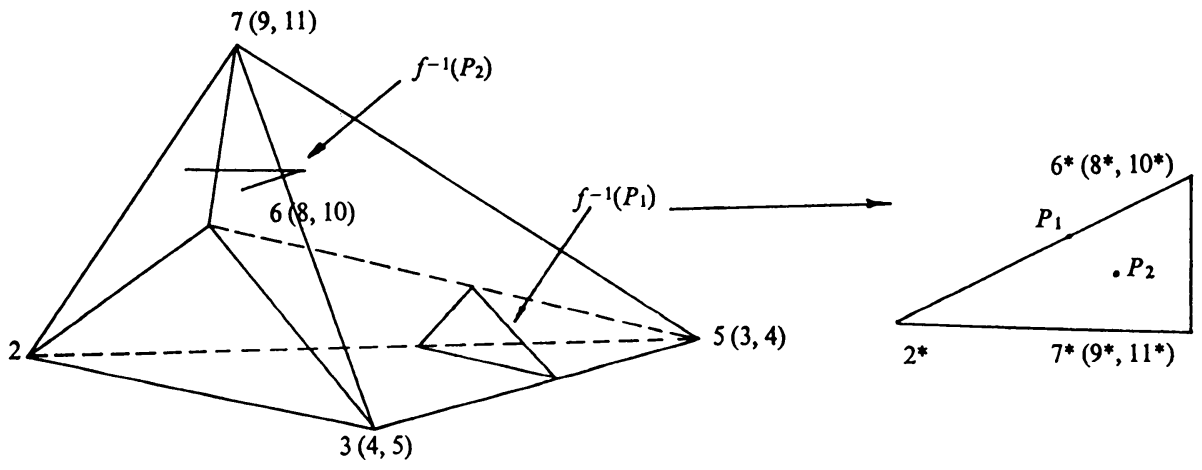

Figure 7. 
is part of a copy of the set denoted by $A_{4}$ in which it is not possible to collapse $(2,5)$ by such a mapping.

This example can be generalized to show that there is a pointlike simplicial mapping from a combinatorial $n$-sphere onto itself which cannot be factored into any nontrivial sequence of simplicial mappings, i.e., identity mappings and $f$ itself. In a slightly different direction Cohen [6] has given an example of a pointlike simplicial mapping of $S^{n}(n \geqq 5)$ onto a complex which is not a combinatorial manifold.

III. Certain mappings can be factored. A set $M$ is starlike with respect to a point $p$ if for any point $q$ in $M$ the straight line from $p$ to $q$ is contained in $M$.

Condition W. There is a 1 -simplex $\sigma_{1}=\left(v_{1}, \dot{v}_{2}\right)$ such that the St $v_{1}$ can be made starlike with respect to $v_{2}$ by a simplicial homeomorphism of the space onto itself.

It is easy to see that Condition W does not hold for any 1-simplex collapsed by the mapping in the example of §II.

The following theorem is the key to whether or not a pointlike simplicial mapping can be factored. All triangulations are assumed to be combinatorial.

THEOREM 1. A 1-Star collapse mapping can be defined on the $n$-sphere if and only if Condition W holds.

Proof. Suppose Condition W holds. Then there is no loss in assuming the St $v_{1}$ to be starlike with respect to $v_{2}$ for some simplex $\sigma_{1}=\left(v_{1}, v_{2}\right)$. Consider the mapping $g$ which is defined to be the identity on the complement of St $v_{1}$ and is defined on St $v_{1}$ to be the linear extension of the vertex mapping $m$ which is the identity on Bdy St $v_{1}$ and which takes the vertex $v_{1}$ to the image of the vertex $v_{2}$ under $m$. Simplexes of the form $\left(v_{1}, v_{j}\right)$ map to the simplexes of the form $\left(v_{2}, v_{j}\right)$ and these will remain in the same complementary domain of Bdy St $v_{1}$ so that the mapping is well defined. The result of the mapping $g$ is a new triangulation of an $n$-cell of the $n$-sphere. That is, the $n$-cell St $v_{1}$ has been replaced by an $n$-cell with a different triangulation and the same boundary. The mapping is obviously a 1-Star collapse mapping. Notice that $g\left(\sigma_{1}\right)$ is a vertex and St $\sigma_{1}$ is collapsed to an $(n-1)$-cell D. Furthermore on the complement of the star of the 1-simplex $\sigma_{1}$, the mapping is a simplicial homeomorphism.

Now suppose that a 1-Star collapse mapping $g$ can be defined on a 1-simplex $\sigma_{1}=\left(v_{1}, v_{2}\right)$. It will be shown that St $v_{1}$ can be made starlike with respect to $v_{2}$. The image of St $v_{1}$ under the 1-Star collapse mapping $g$ is an $n$-cell (it is simplicially homeomorphic to St $\left.v_{1} \mid \mathrm{St} \sigma^{1}\right)$ which can be made starlike with respect to $g\left(v_{1}\right)$ by a simplicial space homeomorphism, $f$. Then the composition of $g$ and $f$ is a 1-Star collapse, $G$, such that $G\left(\mathrm{St} v_{1}\right)$ is starlike with respect to $G\left(v_{1}\right)$. Since no vertices other than $v_{1}$ and $v_{2}$ are identified by $G$, it is a simplicial homeomorphism on the complement of St $\sigma^{1}$.

A new triangulation of $G\left(\mathrm{St} v_{1}\right)$ will be defined such that the $n$-sphere so obtained 
will be simplicially homeomorphic to the domain of $g$. In this new triangulation St $G\left(v_{1}\right)$ will be starlike with respect to the vertex associated with $v_{2}$.

Let $K$ be an $(n-1)$-sphere in $G\left(S^{n}\right)$ with $G\left(v_{2}\right)$ in one complementary domain and $p$ be a point common to $K$ and the interior of $G\left(\right.$ St $\left.v_{1}\right)$. For a sufficiently small diameter of $K$ each straight line from a vertex on the boundary of $G\left(\mathrm{St} v_{1}\right)$ to $p$ will lie in the interior of $G\left(\mathrm{St} v_{1}\right)$. Let this collection of straight lines define a new triangulation of $G\left(\mathrm{St} v_{1}\right)$. That is, each simplex in $G\left(\mathrm{St} v_{1}\right)$ with the new triangulation is the join of $p$ with a simplex in the boundary of $G\left(\mathrm{St} v_{1}\right)$. Let $m$ denote the vertex mapping from $S^{n}$ to $G\left(S^{n}\right)$ defined by $m\left(v_{i}\right)=G\left(v_{i}\right)$ if $i \neq 1$ and $m\left(v_{1}\right)=p$. Let $h$ be the linear extension of $m$. It is clear that $h$ is a simplicial homeomorphism from $S^{n}$ to $G\left(S^{n}\right)$ and that $h\left(\mathrm{St} v_{1}\right)$ is starlike with respect to $h\left(v_{2}\right)$. This completes the proof of Theorem 1 .

The following results will be needed for the next theorem.

LEMma 1. Suppose $K$ is a polyhedral subset of the interior of a combinatorial $n$-manifold $M$ such that $K$ is cellular in $M$. Then if $N$ is a regular neighborhood of $K$ in $M$ the interior of $N$ is an open $n$-cell.

Proof. Since $K$ is polyhedral it is possible to subdivide so that $K$ is a subcomplex. By Whitehead [2], in the second derived subdivision of this triangulation the simplicial neighborhood of $K$ (the collection of all simplexes having a vertex in $K$ ) is a regular neighborhood, $N$. Further, by the same reference, any two regular neighborhoods are combinatorially equivalent.

Since $K$ is cellular in $M$ it is also cellular in $N$. That is, the function $h$ which maps $K$ to a point and is a homeomorphism on $M \backslash K$ is, of course, a homeomorphism on $N \backslash K$.

It is well known that a cellular decomposition of an $n$-manifold with a finite number of nondegenerate elements has a decomposition space homeomorphic to the original manifold.

Consider what happens to $N$ under $h$. By the preceding remark $h(N)$ is homeomorphic to $N$. Furthermore, the interior of $h(N)$ is the open cone over the boundary of $h(N)$ since all vertices in the interior of $N$ were in $K$. This open cone is locally euclidean at the vertex point $h(K)$ and therefore the open cone is an open $n$-cell [4]. Since this is homeomorphic to the interior of $N$, the proof is complete.

Lemma 2. Suppose $M$ is a subset of $S^{n}$. If for any compact subset, $K$, in the complement of $M$ there is an $n$-cell containing $M$ and contained in the complement of $K$ then $M$ is cellular.

Lemma 3. Suppose $g$ is a simplicial mapping defined on $S^{n}$ and $M=g^{-1}(x)$ for some $x$ in $g\left(S^{n}\right)$.

(a) If $x$ is a vertex, $M$ is a subcomplex.

(b) If $x$ is not a vertex, $M$ is a polyhedral set which contains no vertex.

(c) If $x$ is not a vertex, $M \cap \sigma^{j}(j>0$ by (b)) is at most $(j-1)$-dimensional. 
(d) Suppose $g\left(\sigma^{n}\right)=\tau^{n-1}$. Then

(i) $\sigma^{n}$ has two $(n-1)$-faces $\sigma_{1}^{n-1}$ and $\sigma_{2}^{n-1}$ such that g restricted to $\sigma_{i}^{n-1}$ is one-to-one.

(ii) Every other $(n-1)$-face of $\sigma^{n}$ is mapped to an $(n-2)$-simplex.

(iii) Int $\sigma^{n}$ is decomposed into line intervals each parallel to the 1-simplex whose image is a vertex.

(iv) If $R_{i}$ is a subset of $\sigma_{i}^{n-1}, i=1,2$, such that $g\left(R_{1}\right)=g\left(R_{2}\right)$ then $R_{1}$ is homeomorphic to $R_{2}$.

These are well-known results with straightforward proofs. Notice that Lemma 3 says that if $M$ contains interior points of a simplex, $\sigma$, and a vertex then $M$ must contain $\sigma$.

The idea of a Whitehead collapse will also be used. This is defined and discussed in [2] where the next result also appears as Lemma 1 of that paper.

LemmA 4. If $K \cap L_{0} \subset L_{q}$ and $L_{0}$ contracts into $L_{q}$, then $K \cup L_{0}$ contracts into $K \cup L_{q}$.

The following result due to Zeeman and McMillan will also be needed.

Lemma 5. If $X$ contracts or expands to $Y$ and if $X$ is pointlike then $Y$ is also.

This is a special case of Lemma 1 of [3].

THEOREM 2. Suppose $f$ is a pointlike simplicial mapping of $S^{n}$ and $L$ is a nondegenerate inverse image of some vertex of $f\left(S^{n}\right)$. If $g$ is a 1-Star collapse mapping defined on a 1-simplex $\sigma^{1}=\left(v_{1}, v_{2}\right)$ in $L$ then there is a pointlike simplicial mapping $h$ from $g\left(S^{n}\right)$ to $f\left(S^{n}\right)$ such that there is a vertex $p$ in $f\left(S^{n}\right)$ such that $h^{-1}(p)=g(L)$.

Proof. Define the mapping $h$ as $f g^{-1}$.

To show that $h$ is pointlike suppose $x$ is a point of $h\left[g\left(S^{n}\right)\right]$. It must be shown that $h^{-1}(x)$ is a pointlike set. Since $h^{-1}(x)=g f^{-1}(x)$ and $f$ is a pointlike mapping (so that $f^{-1}(x)$ is a pointlike set) it must be shown that $g$ preserves the pointlike character of $f^{-1}(x)$.

Let $M$ denote $f^{-1}(x)$. Then there are four possibilities for $M$ :

(i) $M \cap$ Int St $v_{i}=\square$ where $v_{i}$ is a vertex of $\sigma^{1}$;

(ii) $M \cap$ Int St $v_{i} \neq \square, M \cap$ Int St $\sigma^{1}=\square$;

(iii) $M=L$;

(iv) $M \neq L, M \cap$ Int St $\sigma^{1} \neq \square$.

(i) Suppose $M$ does not intersect the interior of St $v_{i}$, where $v_{i}$ is a vertex of $\sigma^{1}$. Then from the second part of the proof of Theorem 1, the complement of $M$ is homeomorphic to the complement of $g(M)$. Therefore $g(M)$ is pointlike.

(ii) Suppose $M \cap$ Int St $v_{i} \neq \square$ and $M \cap$ Int St $\sigma^{1}=\square$. Let $K$ be a compact set in the complement of $g(M)$. Then $g^{-1}(K)$ is compact in the complement of $M$. Since $M$ is cellular, a cell $C$ can be found which contains $M$, does not intersect Int St $\sigma^{1}$ and is in the complement of $g^{-1}(K)$. Then $g$ is a homeomorphism on $C$ and $g(C)$ is a cell containing $g(M)$ which misses $K$. Therefore by Lemma $2, g(M)$ is cellular and this is equivalent to being pointlike in $S^{n}$. 
(iii) Suppose $M$ is $L$. Then it must be shown that $g(L)$ is pointlike. This will be accomplished in five steps.

(a) First it will be shown that $L \cup$ St $\sigma^{1}$ contracts to $L$ in the sense of Whitehead [2].

If St $\sigma^{1} \subseteq L$ there is nothing to prove.

Suppose St $\sigma^{1} \nsubseteq L$. Let $B=\operatorname{link}\left(\sigma^{1}, S^{n}\right)$ and $A=B \cap L$. Then St $\sigma^{1}=\sigma^{1} \cdot B$ and this contracts to $\sigma^{1} \cdot A$ by Lemma 2, $\$ 3$ of [2]. Since St $\sigma^{1}$ contracts to $\sigma^{1} \cdot A$ and $L \cap S t \sigma^{1} \subseteq \sigma^{1} \cdot A$ then by Lemma $4, L \cup$ St $\sigma^{1}$ contracts to $L \cup \sigma^{1} \cdot A=L$ and accomplishes (a).

(b) Since $L \cup \operatorname{St} \sigma^{1}$ contracts to $L$ and $L$ is pointlike then by Lemma 5 , $L \cup S t \sigma^{1}$ is pointlike.

(c) The set $g\left(L \cup\right.$ St $\left.\sigma^{1}\right)$ is pointlike. To see this consider the following. There is a sequence of $n$-cells $\left\{C_{i}\right\}$ such that $C_{i+1}$ is contained in the interior of $C_{i}$ and $L \cup \mathrm{St} \sigma^{1}$ is the intersection of the sequence. From the proof of Theorem 1 the mapping $g$ is a homeomorphism on the boundary of each $C_{i}$. Therefore $g\left(\operatorname{Bdy} C_{i}\right)$ is an $(n-1)$-sphere in the $n$-sphere. Hence the image under the mapping $g$ of $C_{i}$ is an $n$-cell which contains $g\left(L \cup\right.$ St $\left.\sigma^{1}\right)$. It also follows that the intersection of the sequence $\left\{g\left(C_{i}\right)\right\}$ is $g\left(L \cup \mathrm{St} \sigma^{1}\right)$ by a point-set argument. Hence $g\left(L \cup \mathrm{St} \sigma^{1}\right)$ is cellular and therefore pointlike.

(d) The set $g\left(L \cup\right.$ St $\left.\sigma^{1}\right)$ contracts to $g(L)$. As above in (a) if $g\left(\right.$ St $\left.\sigma^{1}\right) \subseteq g(L)$ there is nothing to prove. Otherwise, let $\sigma^{k}$ be in $g\left(\mathrm{St} \sigma^{1}\right)$ but not in $g(L)$. Then $\sigma^{k}=g\left(\sigma^{1}\right) \cdot \sigma^{k-1}$ where $\sigma^{k-1}$ is not in $L$. This contracts to $g\left(\sigma^{1}\right) \cdot$ Bdy $\sigma^{k-1}$ which consists of $(k-1)$-simplexes of the form $g\left(\sigma^{1}\right) \cdot \sigma^{k-2}$. If $\sigma^{k-2}$ is in $L$ then so is $g\left(\sigma^{1}\right) \cdot \sigma^{k-2}$. If $\sigma^{k-2}$ is not in $L$ then $g\left(\sigma^{1}\right) \cdot \sigma^{k-2}$ is not in $L$ and contracts to $g\left(\sigma^{1}\right) \cdot$ Bdy $\sigma^{k-2}$. Repeating this procedure proves that $\sigma^{k}$ contracts to those faces of $\sigma^{k} g(L)$. That is, $g\left(\right.$ St $\left.\sigma^{1}\right)$ contracts to $g\left(\sigma^{1}\right) \cdot B$ where $B=g(L) \cap g\left[\operatorname{link}\left(\sigma^{1}, S^{n}\right)\right]$. Again Lemma 4 applies to give that $g(L) \cup g\left(\mathrm{St} \sigma^{1}\right)$ contracts to $g(L) \cup g\left(\sigma^{1}\right) \cdot B=g(L)$ and (d) is proved.

(e) Since $g\left(L \cup\right.$ St $\left.\sigma^{1}\right)$ is pointlike and contracts to $g(L)$ then $g(L)$ is pointlike by Lemma 5 .

(iv) Suppose $M$ is not $L$ but does intersect the interior of St $\sigma^{1}$. Note that in this case $M$ contains no vertex by Lemma 3(b).

Suppose $K$ is a compact set in the complement of $g(M)$.

The outline of the proof is as follows: A description will be given of a first and second derived subdivision of $S^{n}$. It will be shown that, $N_{2}$, the interior of the simplicial neighborhood of $M$ in the second derived is an open $n$-cell which maps to an open $n$-cell under $g$. Since $g(M)$ is contained in the open $n$-cell $g\left(N_{2}\right)$ it is also contained in an $n$-cell in the interior of $g\left(N_{2}\right)$. Hence there is an $n$-cell containing $g(M)$ and missing $K$. Therefore by Lemma $2, g(M)$ is pointlike.

The set $g^{-1}(K)$ is compact and in the complement of $M$. Let $\varepsilon>0$ be small enough that the $\varepsilon$-neighborhood of $M$, (using the linear metric) $N(M, \varepsilon)$, misses $g^{-1}(K)$ and intersects no simplex that $M$ does not. (See Lemma 3.) 
Prior to describing the derived subdivisions some observations and notation will be helpful.

First, $N(M, \varepsilon) \cap$ Bdy St $\sigma^{1}$ consists of $2 k$ components $C_{i j}, i=1,2, j=1, \ldots, k$, where $C_{1 j}$ is homeomorphic to $C_{2 j}$. This follows directly from Lemma 3(d).

Second, St $\sigma^{1} \cap \operatorname{Bdy} N(M, \varepsilon)$ consists of a union of line intervals each parallel to $\sigma^{1}$ with one endpoint in $\bar{C}_{1 j}$ and the other endpoint in $\bar{C}_{2 j}$ for some $j$.

Finally, if $M \cap \sigma^{j} \neq \square$ then $N(M, \varepsilon) \cap \sigma^{j}$ is $j$-dimensional so that Bdy $N(M, \varepsilon)$ is no more than $(j-1)$-dimensional.

Obtain the vertices for a first derived subdivision in the following way: If $M \cap$ Int $\sigma^{j}=\square$ use the barycenter of $\sigma^{j}$ as a new vertex. If $M \cap$ Int $\sigma^{j} \neq \square$ use, instead of the barycenter, any point in $M \cap$ Int $\sigma^{j}$. In more detail the process is as follows: First, $M$ contains no vertex of the original triangulation by Lemma 3(b). By Lemma 3(c) $M$ can contain at most one interior point of a 1-simplex. Use this point as a vertex instead of the barycenter of the 1-simplex. If $M$ intersects a 2-simplex the intersection is either a point, $p$, or a line segment by Lemma 3(c). Instead of the barycenter of the 2-simplex use $p$ if the intersection is just $p$; otherwise use any point on the line interval. This process can be repeated in each dimension to obtain a well-defined first derived subdivision. In this triangulation $M$ is a subcomplex.

Obtain the vertices for a second derived subdivision in the following manner: If $\sigma^{j}$ is a simplex in the first derived subdivision which does not intersect St $\sigma^{1} \cap \operatorname{Bdy} N(M, \varepsilon)$, where St $\sigma^{1}$ is the point set from the original triangulation, use the barycenter of $\sigma^{j}$. If $\sigma^{j} \cap\left[\operatorname{St} \sigma^{1} \cap \operatorname{Bdy} N(M, \varepsilon)\right] \neq \square$ use, instead of the barycenter, any point of the intersection. A detailed process, exactly like that for $M$, could be given to describe the selection of the vertices. Let $\mathrm{N}_{2}$ denote the interior of the simplicial neighborhood of $M$ in the second derived subdivision if it does not intersect $g^{-1}(K)$. It certainly does not intersect $g^{-1}(K)$ in St $\sigma^{1}$. If it does in $S^{n} \mid S t \sigma^{1}$ it is possible to subdivide $S^{n} \mid S t \sigma^{1}$ in such a way that no vertices are added in $M$ but after sufficient subdivision the simplicial neighborhood of $M$ does miss $g^{-1}(K)$. In this case let $N_{2}$ denote the interior of the regular neighborhood so obtained. By Lemma $1, N_{2}$ is an open $n$-cell. It contains $M$ and misses $g^{-1}(K)$.

Consider $N_{2} \cap$ St $\sigma^{1}$. This consists of $k$ components $P_{j}, j=1, \ldots, k$. Each $P_{j}$ intersects Bdy St $\sigma^{1}$ in homeomorphic components $C_{i j}, i=1,2$. (See Lemma 3.)

Each $C_{i j}$ is collared in $N_{2}$. That is, there is a homeomorphism $h_{1 j}$ which maps $C_{1 j} \times[0,1 / 4]$ into $N_{2}$ taking $C_{1 j} \times\{1 / 8\}$ onto $C_{1 j}$ and a homeomorphism $h_{2 j}$ which maps $C_{2 j} \times[3 / 4,1]$ into $N_{2}$ taking $C_{2 j} \times\{7 / 8\}$ onto $C_{2 j}$. Let $R_{1 j}=h_{1 j}\left(C_{1 j} \times[0,1 / 4]\right)$ and $R_{2 j}=h_{2 j}\left(C_{2 j} \times[3 / 4,1]\right)$. The homeomorphisms are taken so that $R_{1 j} \cap R_{2 j}=\square$.

Each $P_{j}$ is homeomorphic to $C_{1 j} \times[0,1]$ and hence there is a homeomorphism $h_{j}$ which maps $C_{1 j} \times(1 / 4,3 / 4)$ onto $P_{j} \mid\left(R_{1 j} \cup R_{2 j}\right)$. Consider

$$
g\left(R_{1 j} \cup P_{j} \cup R_{2 j}\right)=g\left(R_{1 j}\right) \cup g\left(P_{j}\right) \cup g\left(R_{2 j}\right) .
$$

Since $g\left(P_{j}\right)$ is homeomorphic to $C_{1 j} \times\{t\}, g\left(R_{1 j}\right)$ is homeomorphic to $C_{1 j} \times[1 / 8,1 / 4]$ 
and $g\left(R_{2 j}\right)$ is homeomorphic to $C_{2 j} \times[7 / 8,1]$ the union is homeomorphic to $C_{1 j} \times[0,1]$. (Recall that $C_{1 j}$ is homeomorphic to $C_{2 j}$.) Denote the homeomorphism by $\hat{h}_{j}$, i.e., $\hat{h}_{j}\left[g\left(R_{1 j} \cup P_{j} \cup R_{2 j}\right)\right]=C_{1 j} \times[0,1]$.

Define

$$
\begin{aligned}
h(x) & =g(x) & & \text { if } x \in N_{2}, \quad x \notin R_{1 j} \cup P_{j} \cup R_{2 j} \quad \text { for any } j, \\
& =\hat{h}_{j}^{-1}\left(h_{i j}^{-1}(x)\right) & & \text { if } x \in R_{i j}, \\
& =\hat{h}_{j}^{-1}\left(h_{j}^{-1}(x)\right) & & \text { if } x \in P_{j} \mid\left(R_{1 j} \cup R_{2 j}\right) .
\end{aligned}
$$

Then $h$ is a homeomorphism from $N_{2}$ onto $g\left(N_{2}\right)$. Hence $g\left(N_{2}\right)$ is an open $n$-cell and the proof for case (iv) is complete.

With reference to case (iv) there are some interesting alternatives. First, for $n=3$ it is easy to see how to consider all the possibilities for $M \cap \mathrm{St} \sigma^{1}$, select polyhedral cells closing on $M$ and then "push" these around to make their intersection with St $\sigma^{1}$ parallel to $\sigma^{1}$ so that $g$ would map them to cells closing on $g(M)$ and therefore prove that $g(M)$ is cellular.

An alternate proof of (iv) for $n \geqq 5$ can be based on the following result of McMillan (Theorem 1 of [5]).

LEMMA 6. Let $X$ be a compact absolute retract in the interior of a piecewise-linear manifold $M^{n}, n \geqq 5$. Then, a necessary and sufficient condition that $X$ be cellular with respect to piecewise-linear cells is that the following property hold:

For each open set $U$ containing $X$, there exists an open set $V$ such that $X \subset V \subset U$ and each loop in $V \backslash X$ is null-homotopic in $U \backslash X$.

Let $U$ be any open set containing $g(M)$. Then $g^{-1}(U)$ is an open set containing $M$. Since $M$ is cellular, by Lemma 6 there is an open set $W$ containing $M$ and contained in $g^{-1}(U)$ such that any loop in $W \backslash M$ is null-homotopic in $g^{-1}(U) \backslash M$.

Let $\varepsilon>0$ be small enough so that the $\varepsilon$-neighborhood of $M, N(M, \varepsilon)$, is included in $W$.

Let $V=g(\operatorname{Int} N(M, \varepsilon))$. Then $V$ is open. To see this let $p \in V$. Then $g^{-1}(p)$ is either a point or an interval parallel to $\sigma^{1}$. If $g^{-1}(p)$ is a point then there is an open set about $p$ contained in $N(M, \varepsilon)$ on which $g$ is a homeomorphism (i.e., it misses St $\left.\sigma^{1}\right)$ so $p$ is contained in an open set in $V$. If $g^{-1}(p)$ is an interval then there is an open cylinder about the interval contained in $N(M, \varepsilon)$ such that the image of the cylinder under $g$ is an open set containing $p$ and, of course, contained in $V$. Hence $V$ is open.

Clearly $V$ contains $g(M)$ and is contained in $U$.

It will be shown that any loop in $V \mid g(M)$ is null-homotopic in $U \mid g(M)$. Then by Lemma 6 this will imply that $g(M)$ is cellular. Suppose $L$ is a loop in $V \backslash g(M)$. Consider $g^{-1}(L)$. This is a set in $W$ which consists of a homeomorphic copy of $L$, say $\hat{L}$, with possibly some subset crossed with an interval. This is true since the inverse image of a point is either a point or an interval. In any case $g^{-1}(L)$ is homotopic to $\hat{L}$ by a homotopy $f_{t}^{\prime}, t \in[0,1]\left(f_{1}^{\prime}\left[g^{-1}(L)\right]=\hat{L}\right)$. 
Since $M$ is cellular $\hat{L}$ is null-homotopic in $g^{-1}(u) \backslash M$ by the choice of $W$. Let $f_{t}^{\prime \prime}, t \in[0,1]$, denote the homotopy which shrinks $\hat{L}$ to a point.

Define, for $y$ in $L$,

$$
f_{t}(y)=g\left[f_{t}^{\prime \prime}\left(f_{1}^{\prime}\left(g^{-1}(y)\right)\right)\right] .
$$

This is a homotopy which shrinks $L$ to a point in $U \backslash M$. Therefore $g(M)$ is cellular.

This completes the proof that $h$ is a pointlike mapping.

It remains to show that $h$ is a simplicial mapping. Suppose $\tau$ is in $g\left(S^{n}\right)$. Then $\tau$ either intersects the interior of the $(n-1)$-cell $C^{\prime}$ which is the image of St $\sigma^{1}$ or not.

If not, then $\tau$ is simplicially homeomorphic to $g^{-1}(\tau)$ and $f$ is simplicial. Therefore $h$ is a simplicial mapping on the complement of the interior of the $(n-1)$-cell $C^{\prime}$.

If, however, $\tau$ intersects the interior of $C^{\prime}$ then $\tau$ is a $j$-simplex and $g^{-1}(\tau)$ is a $(j+1)$-simplex. In any case $\tau$ is simplicially homeomorphic to a face of $g^{-1}(\tau)$. Then $f$ maps this face simplicially to a simplex whose dimension is no greater than that of $\tau$. Hence $h=f g^{-1}$ is simplicial on $\tau$.

This completes the proof of Theorem 2.

THEOREM 3. Suppose $f$ is a pointlike simplicial mapping of the $n$-sphere. Then $f$ can be factored into a 1-Star collapse mapping $f_{1}$ and a pointlike simplicial mapping $f_{2}$ if and only if Condition $\mathrm{W}$ holds for a 1-simplex collapsed by $f$.

Proof. Suppose Condition $\mathrm{W}$ holds for a 1-simplex collapsed by $f$. Let $F=f_{1} f_{2}$ where $f_{1}$ and $f_{2}$ are the mappings which exist by Theorems 1 and 2 . It must be shown that $F\left(S^{n}\right)$ is simplicially homeomorphic to $f\left(S^{n}\right)$. To see this consider the following. Let $m$ be the vertex mapping of $F\left(S^{n}\right)$ to $f\left(S^{n}\right)$ defined by

$$
m(w)=f\left[F^{-1}(w)\right] .
$$

If $\sigma^{n}=\left(w_{0}, \ldots, w_{n}\right)$ is an $n$-simplex of $F\left(S^{n}\right)$ then $F^{-1}\left(\sigma^{n}\right)$ is a complex which has at least one vertex in each $F^{-1}\left(w_{i}\right)$, which has no vertex outside $\bigcup_{i=0}^{n} F^{-1}\left(w_{i}\right)$ and which contains exactly one $n$-simplex on which $F$ is one-to-one. Then $f\left[F^{-1}\left(\sigma^{n}\right)\right]$ is an $n$-simplex of $f\left(S^{n}\right)$. Let $H$ be the linear extension of the mapping $m$. Then $H$ is a simplicial homeomorphism.

Conversely, if Condition $\mathrm{W}$ does not hold by Theorem 1 it is not possible to define a 1-Star collapse and therefore not possible to factor as indicated.

This completes the proof of Theorem 3.

Obviously, the process described by the theorem can be continued until some $f$ is obtained such that Condition $\mathrm{W}$ does not hold. Hence complete factorization may be possible.

In considering a pointlike simplicial mapping $f$ defined on $S^{n}$, Finney's theorem states that for $n=3, f\left(S^{3}\right)=S^{3}$. No such assumption was made above for $n \neq 3$. However, on the basis of the preceding theorem, a theorem analogous to Finney's for $n \geqq 3$ does follow. 
THEOREM 4. If $f$ is a pointlike simplicial mapping of $S^{n}, n \geqq 3$, which can be completely factored into a sequence of 1-Star collapse mappings, then $f\left(S^{n}\right)=S^{n}$.

Proof. $f_{n} f_{n-1} \cdots f_{1}\left(S^{n}\right)$ is a triangulated $n$-sphere since each $f_{i}$ maps $S^{n}$ onto itself and by Theorem 2 is simplicially homeomorphic to $f\left(S^{n}\right)$.

IV. Factoring in subdivisions. The purpose of this section is to answer two questions which arise in connection with the example in §II. The first is "Do there exist subdivisions of the domain and range relative to which the mapping $f$ defined in $\S \mathrm{II}$ is simplicial and such that $f$ can be factored into a sequence of 1-Star collapse mappings?". Since the subcomplex which is mapped to a point in the example is collapsible (it is a disk) the second question is "If $C$ is a subcomplex which is not collapsible (e.g., Bing's "house with two rooms") and $f$ is a pointlike simplicial mapping such that $C=f^{-1}(v)$ for some vertex $v$ in the range do there exist subdivisions relative to which $f$ is simplicial and such that $f$ can be factored into a sequence of 1-Star collapse mappings?"

The answer to the first question is affirmative and Figure 8 indicates how to

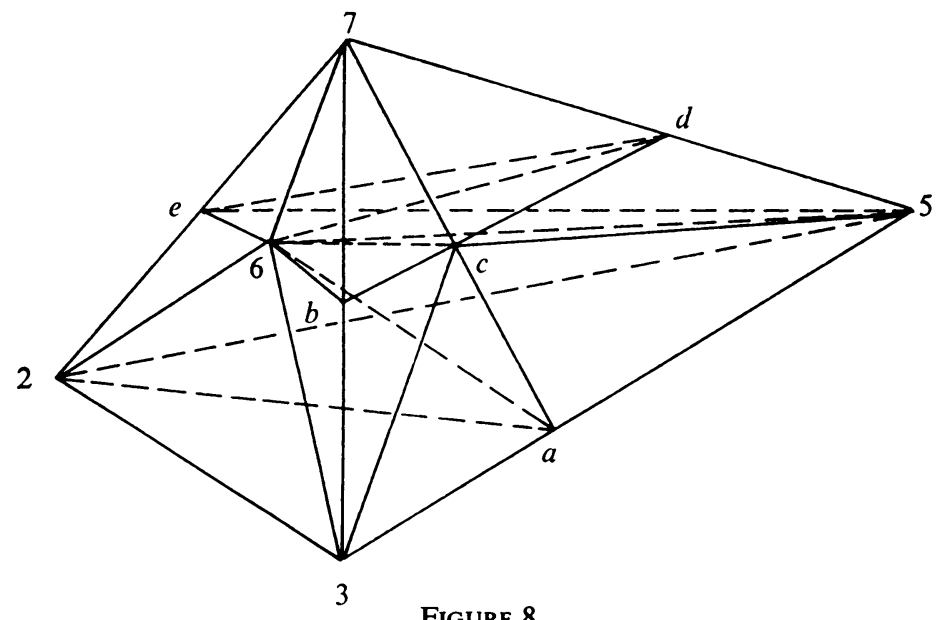

make the subdivision. Of course, the motivation of the subdivision is to force each 1 -simplex collapsed by $f$ to satisfy Condition W. The subdivision on the remainder of the domain is that induced by the vertices shown, i.e., no other vertices are to be added. The subdivision of the range is also that induced by the images of these vertices. Further $b, c, d$, and $e$ are chosen such that $f(b)=f(c)=f(d)=f(e)$.

The 1-simplexes should be collapsed as follows: (1) $(a, 2)(2)(2,3)(3)(2,4)$ (4) $(2,5)(5)(b, c)(6)(c, d)(7)(e, d)$. It is not difficult to verify the validity of this sequence, i.e., each is a 1-Star collapse and the product has the same effect as $f$. One observation serves to answer the second question.

LEMMA 7. Suppose $C$ is a 2-complex in $S^{3}$ which has no free edges. If $\sigma^{1}$ is a 1-simplex of $C$ which satisfies Condition $\mathrm{W}$ and $g$ is a 1-Star collapse defined on $\sigma^{1}$ then $g(C)$ has no free edges. 
Proof. Let $v$ be a vertex of $\sigma^{1}$ such that St $v$ is starlike with respect to the other vertex of $\sigma^{1}$. If $\sigma^{2} \subset C$ has $v$ for a vertex then there are two other 2-simplexes in $C$ each having $v$ for a vertex and each sharing a 1-simplex with $\sigma^{2}$ since $C$ has no free edges. Hence $C \cap \mathrm{St} v$ is a disk $D$ with the vertex $v$ interior and consisting of 2 -simplexes. Furthermore each 1-simplex in the boundary of $D$ is the common edge of a 2-simplex in $D$ and a 2-simplex of $C$ not in $D$. Consider the effect on $C$ of the mapping $g$. Outside the interior of $D, g$ can be taken as the identity. (Recall the proof of Theorem 1.) The 2-simplexes in $D \cap S t \sigma^{1}$ are mapped to 1 -simplexes on the boundary of $D$. On $D \backslash S t \sigma^{1}$ the mapping is a simplicial homeomorphism. Therefore if $\tau$ is a 1-simplex in $g(C)$ it is either

(a) outside $g(\operatorname{Int} D)$, in which case it is in still incident with two 2-simplexes of $g(C)$ or

(b) a 1-simplex of the form $(g(v), t)$ where $t$ is a vertex on the boundary of $D$, in which case it is the simplicially homeomorphic image of $(v, t)$ which is the common side of two 2-simplexes of $C$.

Therefore $g(C)$ has no free edges.

This lemma answers the question because even if subdivisions are made to allow collapses it will never be possible by 1-Star collapses to even reduce the dimension of $C$ and certainly not map it to a point.

The author wishes to acknowledge the helpful comments and suggestions made by the referee and to thank him in particular for raising the questions in §IV. A major part of this paper is part of a dissertation written under Dr. Lida Barrett, to whom the author is indebted. Also much benefit was derived from past association with Professor O. G. Harrold and from discussion with L. C. Glaser.

\section{BIBLIOGRAPHY}

1. Ross Finney, Pointlike simplicial mappings of a 3-sphere, Canad. J. Math. 15 (1963), 591-604.

2. J. H. C. Whitehead, Simplicial spaces, nuclei and m-groups, Proc. London Math. Soc. (2) 45 (1939), 243-327.

3. D. R. McMillan, Jr. and E. C. Zeeman, On contractible open manifolds, Proc. Cambridge Philos. Soc. 58 (1962), 221-224.

4. Ronald H. Rosen, Stellar neighborhoods in polyhedral manifolds, Proc. Amer. Math. Soc. 14 (1963), 401-406.

5. D. R. McMillan, Jr., A criterion for cellularity, Ann. of Math. 79 (1964), 327-337.

6. Marshall M. Cohen, Simplicial structures and transverse cellularity, Ann. of Math. 85 (1967), 218-245.

\section{UNIVERSITY OF TENNESSEE, KNOXVILLE, TenNessee \\ UNIVERSITY OF HOUSTON, Houston, TeXas}

